\title{
DESIGN OF THE CONTROL SYSTEM FOR THE 1.8-GEV THIRD-GENERATION SYNCHROTRON-RADIATION SOURCE AT TSRF
}

\author{
Noriichi KANAYA ${ }^{\#}$, Shoji SUZUKI'), Shigeru SATO*) and Masahiro KATOH \\ Photon Factory \\ High Energy Accelerator Research Organization (KEK) \\ 1-1, Oho, Tsukuba, 305-0801, Japan \\ *) Department of Physics, Graduate School of Science, Tohoku University, \\ Aobaku, Sendai, 980-8578,Japan
}

\begin{abstract}
A control system has been designed for the $1.8-\mathrm{GeV}$ synchrotron-radiation source at Tohoku University Synchrotron Radiation Facility (TSRF), Sendai-city, Japan. TSRF is a third-generation synchrotron-radiation facility proposed at Tohoku University, Sendai, Japan. The control system comprises three physical layers: UNIX workstations, an FDDI (Fiber Distributed Data Interface) high-speed network, and VME modules. UNIX workstations are employed for the upper layer that provides process control and a user interface. The FDDI provides a 100-Mbps, token-passing, dual-ring LAN using a fiber-optic link suitable for reliably exchanging control data. On the bottom layer, there are VME crates with high performance CPUs that are interconnected to the magnets, RF, vacuum, beamposition monitors, wigglers/undulators, beamlines and related components of the storage ring for local process control. The design of the TSRF control system is discussed.
\end{abstract}

\section{INTRODUCTION}

TSRF (Tohoku-university Synchrotron Radiation Source Facility) is a new third-generation synchrotronradiation source that is currently proposed at Tohoku University, Sendai, approximately $350 \mathrm{~km}$ north of Tokyo, Japan. TSRF is planned to be constructed at the site of the Laboratory of Nuclear Science, Tohoku University, where a 300-MeV-Linac and a $1.2-\mathrm{GeV}$ Stretcher Booster Ring are currently in operation for nuclear-physics experiments [1]. By taking advantage of the existing facility, TSRF employs the Stretcher Booster Ring as the injector for the TSRF storage ring. This can greatly reduce the construction cost for the TSRF synchrotron-radiation source.
TSRF is designed to provide VUV-SX synchrotron radiation to the experimental hall where VUV experiments, surface physics, soft x-ray lithography, microscopy and crystal structure analysis, will be simultaneously carried out.

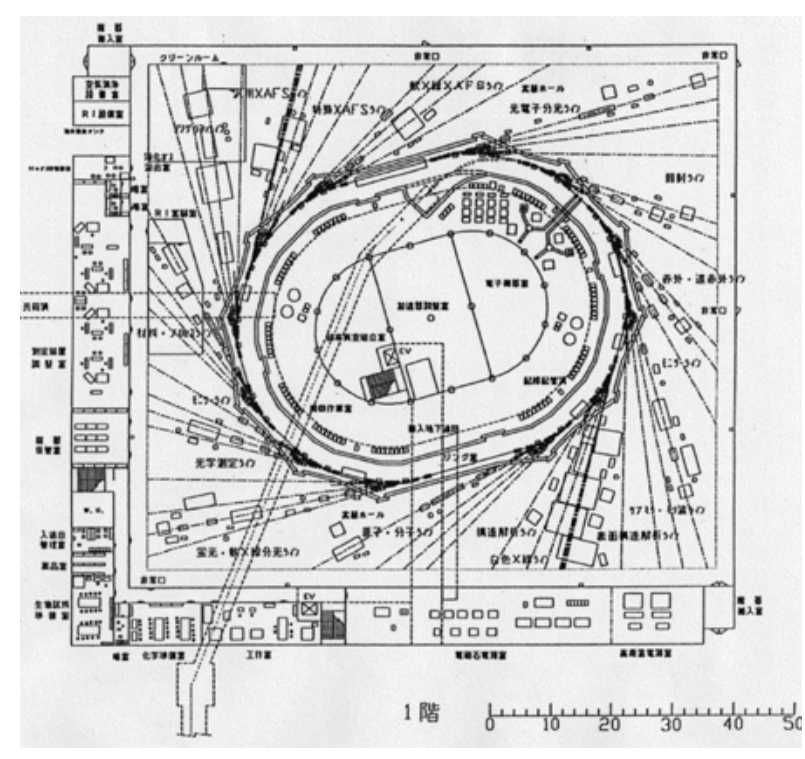

Fig.1 Plan view of the 1.8-GeV TSRF synchrotronradiation storage ring at Tohoku University, Sendai, Japan

The high-power wiggler/undulator beam lines are simultaneously in operation, producing very intense synchrotron-radiation beams [2]. The high-power beam lines are distributed along the long circumference of the storage ring. TSRF has a $1.8-\mathrm{GeV}$ storage ring with a DBA (double-bend-achromat) type, third-generation storage ring with emittance of $4.9 \mathrm{~nm} \mathrm{rad}$, and a

${ }^{\#}$ Email: noriichi.kanaya@kek.jp 
circumference of $244 \mathrm{~m}$. TSRF has more than ten wigglers/undulators, and thirty beamlines for soft X-ray and VUV experiments for research. Figure 1 shows a plan view of the TSRF facility, which includes the 1.2GeV Stretcher Booster Ring.

The control system has been designed for the 1.8$\mathrm{GeV}$ TSRF synchrotron-radiation source in order to operate the storage ring, providing stable synchrotron radiation to users at the experimental hall, and to improve the performance of the storage ring. In this paper, the design of the TSRF control system is discussed.

\section{SYSTEM CONFIGURATION}

The control system provides two major operating modes at the users' request: multi-bunch beam operation and single-bunch beam operation for time-resolved experiments. In addition, the control system allows an operator to provide both (1) automatic setup-and-go operation of the accelerator for users' experiments and (2) machine-study operation with remote control of the accelerator system in order to improve the accelerator performance and to study accelerator physics.

Figure 2 shows the hierarchy of the software system which comprises three layers based on a client-server model. On the top layer, there are Console Managers as clients for operator console management. At the bottom layer, there are Device Servers, which actually interface between the physical hardware devices and the servers at the upper layers. A Dispatcher is a server in the middle layer, which resides among Console Managers and Device Servers at the lowest level.

PCs are cost-effective equipment as operator consoles running at the top layer. Five PCs running under WindowsNT are utilized as console devices with a 20inch bit-map color display, and a trackball used as a pointing device; they are also connected to the network. Under normal operation, two consoles are assigned to storage-ring control, and the other to the beamtransport-line, injection-pulse magnets, safety interlock system and the beamline control system. Man-machine interfaces, including graphics status displays, provide pull-down and pop-up menus, which are organized in a tree structure. Each branch menu is utilized to specify a device and its parameters. The Console Manager always depicts the present status of the accelerator complex.

There are five UNIX workstations at the middle layer for managing VME crates that are utilized for process control. Automatic operation is to setup, in principle, the parameters to devices, such as the bending magnets and families of quadrupole magnets, and then to initiate them. This can be carried out by invoking commands defined in a command file, whose file name is specified by an automatic instruction message solicited by an operator. The workstations automatically carry out the following procedures: (1)Standby-forbeam-injection, (2)Beam-Injection, (3)BeamAcceleration, (4) Re-injection (to set up parameters for injection of the beam after automatically initializing magnets), and (5)Shutdown.

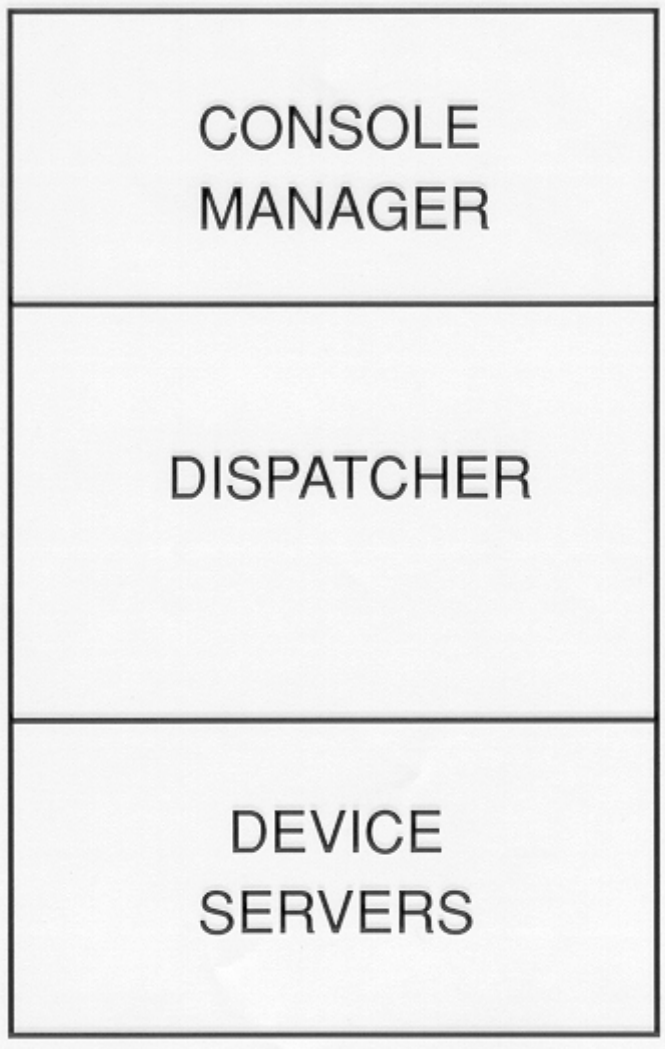

Fig. 2 Hierarchy of the software system

The UNIX workstations, VME crates and PCs are connected to the FDDI (Fiber Distributed Data Interface) high speed network as shown in Fig.3. The FDDI provides a 100-Mbps, token-passing, dual-ring LAN using a fiber-optic link suitable for exchanging control data reliably.

On the bottom layer, there are VME crates with high performance CPUs that are interconnected to the magnets, RF, vacuum, beam-position monitors, wigglers/ undulators, beamlines and related components of the storage ring for local process control. VME modules in the crates carry out time-critical jobs for the accelerator components: during beam acceleration at the storage ring, a power supply for bending magnets of the storage ring, and power supplies for families of 
quadrupole magnets are automatically synchronized in order to keep the betatron values constant.

There is a shared memory table in which all of the operational parameters of the accelerator complex are stored in the relevant data entries. The table is also mapped to the physical memory, which can be referenced by any application process. The purpose of the table is to allow all application processes to utilize the operational parameters as well as the present status of the accelerator complex necessary for their specific operation without directly accessing actual physical devices [3].

There is an on-line database system. The host collects all operational data and control data of the accelerator components. The operational and control data have been automatically stored in the database system to retrieve later specific combinations of behaviors of the accelerator components.

The storage ring has a number of components and sensors to be controlled, including vacuum valves, interlock devices, cooling water flow sensors, fast/slow vacuum sensors, pneumatic-pressure sensors, atmosphere sensors, open-request signals from the storage ring /the experimental hall, vacuum pressure gauges of the storage ring and the beam lines, valve driving units. These components are controlled and monitored by a computer of the control system.

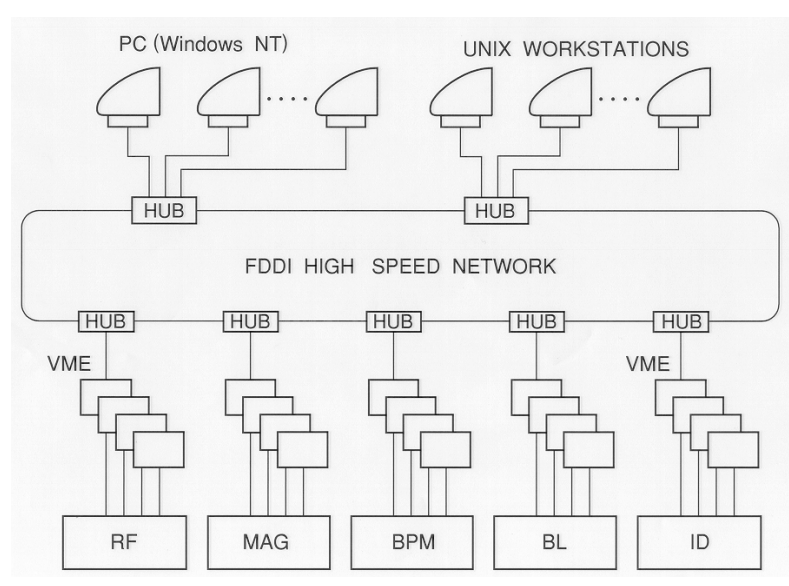

Fig.3 Configuration of the control system

\section{CONCLUSION}

The design of the control system is discussed for the 1.8-GeV third-generation synchrotron-radiation facility TSRF [4], Sendai-city, where a $300-\mathrm{MeV}$ Linac and $1.2-\mathrm{GeV}$ Stretcher Booster Ring are ready as the injector for the $1.8-\mathrm{GeV}$ storage ring. The control system comprises three layers, and is composed of workstations, VME crates and PCs, which are connected to the high-speed FDDI network. The control system provides automatic operation for users' experiments as well as machine-study operation to improve the accelerator performance and to study accelerator physics.

\section{ACKNOWLEGEMENTS}

The authors wish to express their gratitude to Prof.J.Kasagi, Laboratory of Nuclear Science (LNS), Prof.M.Watanabe,. Research Institute of Scientific Measurements, Tohoku University for their valuable discussions, the staffs of LNS and all working group members of the project for their assistance and cooperation.

\section{REFERENCES}

[1] M.Oyamada et al.,Proc. of 10th Symp. on Accel. Sci.Tech.p463,1995 (in Japanese).

[2] M.Katoh, S.Sato, S.Suzuki and T.Yamakawa,"Lattice Design of the Synchrotron Radiaiton Source at Tohoku University," Proc. of the 5th European Particle Accelerator Conference,Spain,June, 1996.

[3] N.Kanaya, S.Asaoka and H.Maezawa, "A Generic Model for Monitoring Operational Parameters of Accelerators by Heterogeneous Multiprocesses at the Photon Factory", XIV Int'l Conf. on High Energy Accelerators, July, 1992, Hamburg, Germany.

[4] http://hera.kek.jp/ 